\title{
BMJ Open Impact of using patient-reported outcome measures in routine clinical care of paediatric patients with chronic conditions: a systematic review protocol
}

\author{
Sumedh Bele, ${ }^{1}$ Bijan Mohamed, ${ }^{2}$ Ashton Chugh, ${ }^{2}$ Lotte Haverman, ${ }^{3}$ \\ Maria-Jose Santana ${ }^{1,2}$
}

To cite: Bele S, Mohamed B, Chugh A, et al. Impact of using patient-reported outcome measures in routine clinical care of paediatric patients with chronic conditions: a systematic review protocol. BMJ Open 2019;9:e027354. doi:10.1136/ bmjopen-2018-027354

\section{- Prepublication history and} additional material for this paper are available online. To view these files, please visit the journal online (http://dx.doi. org/10.1136/bmjopen-2018027354).

Received 18 0ctober 2018 Revised 7 February 2019 Accepted 22 February 2019

Check for updates

(C) Author(s) (or their employer(s)) 2019. Re-use permitted under CC BY-NC. No commercial re-use. See rights and permissions. Published by BMJ.

${ }^{1}$ Department of Community Health Sciences, University of Calgary Cumming School of Medicine, Calgary, Alberta, Canada

${ }^{2}$ Department of Pediatrics, University of Calgary Cumming School of Medicine, Calgary, Alberta, Canada

${ }^{3}$ Psychosocial Department, Amsterdam UMC, University of Amsterdam, Amsterdam, The Netherlands

Correspondence to Dr Maria-Jose Santana; mjsantan@ucalgary.ca

\section{ABSTRACT}

Introduction Chronic diseases among children are associated with lower health-related quality of life (HRQOL) and higher utilisation of healthcare services. Integrating Patient-Reported Outcomes Measures (PROMs) in routine clinical care has been shown to reduce utilisation of healthcare services while improving patient outcomes. The objectives of our study are to: (1) identify previously implemented and evaluated PROMs for chronic conditions in paediatric settings; (2) consolidate the evidence to evaluate the impact of using PROMs on HRQOL, healthcare utilisation, patient outcomes (eg, symptoms control) and quality of care among paediatric patients with chronic conditions. The findings from this review will inform the future integration of PROMs in paediatric clinical practice. Methods and analysis We will systematically search the following electronic databases: MEDLINE, EMBASE, CINAHL, PsychINFO and Cochrane library. Reference lists of included studies will also be searched in Web of Science (Thomson Reuters) database to ensure more complete coverage. Two reviewers will independently screen the studies and abstract the data using standardised form. Extracted data will be analysed and synthesised. Finally, a narrative synthesis of summarised data will be presented.

Ethics and dissemination Ethical approval is not required, as the proposed systematic review will use data from published research articles. The results of this study will be disseminated through publication in peer-reviewed journals, scientific conferences and meetings, and the lead author's doctoral dissertation.

PROSPERO registration number CRD42018109035.

\section{INTRODUCTION}

Children with chronic diseases report having a lower health-related quality of life (HRQOL) and higher utilisation of healthcare services compared with their healthy peers; they require complex care. ${ }^{1}$ Higher utilisation of healthcare services, including higher rates of hospitalisation for these populations, poses a challenge for healthcare systems to provide quality care to children with chronic diseases. $^{2} 3$ According to the WHO's definition, chronic diseases are those that are
Strengths and limitations of this study

A key strength of this study is that it is a first systematic review to evaluate the impact of Patientreported Outcome Measures on the Health-related Quality of Life , utilisation of healthcare services, patient outcomes and quality of care among paediatric patients with chronic conditions.

- The findings of this study will provide crucial evidence for integration of PROMs in paediatric clinical care.

- Another strength of this systematic review is that patient-partners will be consulted to assess the face validity of the included studies to verify if the extracted data is meaningful from patients' perspective.

- Exclusion of studies that are not published in English is a potential limitation of this systematic review.

not passed from person to person, they are of long duration and generally have slow progression. ${ }^{4}$

Patient-reported outcome (PRO) are defined as 'the measurement of any aspect of a patient's health status that comes directly from the patient (ie, without the interpretation of the patient's responses by a physician or anyone else), ${ }^{5}$ Patient-reported outcome measures (PROMs) are the tools or instruments used to measure PROs. Evidence from adult populations suggests that the integration of PROMs in clinical care enhances patient-clinician communication by increasing the frequency of discussion of patient outcomes during consultations. ${ }^{6}$ Among patients with metastatic cancer, integration of PROMs in routine clinical care was associated with increased survival compared with usual care, ${ }^{7}$ among patients with arthritis, it improved self-perceived health and disease activity. ${ }^{8}$ It also reduces the use of healthcare services by improving symptom control, increasing patient satisfaction ultimately improves HRQOL. ${ }^{6-12}$ PROMs are 
mostly self-completed questionnaires that measure the patient's health status by asking them about outcomes, such as their symptoms and aspects that may be affected by the disease(s) and/or treatment, including physical, psychological, social, overall well-being and HRQOL. ${ }^{51314}$ PROMs are standardised and validated questionnaires that are either generic or condition-specific. Generic PROMs can be used for all patients which allows comparison of outcomes between different patient groups. ${ }^{15}$ On the other hand, condition-specific PROMs are used for specific conditions, making it more sensitive to outcomes associated with that particular condition. This systematic review aims to identify previously implemented and evaluated generic or condition-specific PROMs in paediatric settings including home, community, outpatient and inpatient healthcare settings.

PROMs also generate 'patient-centred data', so PROMs are increasingly being used as organisational performance measures by clinicians and healthcare administrators to enhance the quality of care. ${ }^{16}{ }^{17}$ Although PROMs are being used to assess the effectiveness of new treatment regimens or surgical procedures, or improve quality of care, evidence around their effectiveness in paediatric clinical care are still scarce. ${ }^{18}$ Due to the scarcity of evidence, they have not been systematically integrated into clinical care. ${ }^{19}{ }^{20}$ To fill this evidence gap, the current systematic review aims to evaluate the impact of PROMs on HRQOL, utilisation of healthcare services, patient outcomes and quality of care among paediatric patients with chronic conditions.

\section{Health-related quality of life}

According to the WHO, HRQOL is 'individual's perception of their position in life in the context of the culture and value systems in which they live and in relation to their goals, expectations, standards and concerns' ${ }^{21}$ Chronic conditions pose a greater risk for psychosocial issues among children, ${ }^{22}$ so the use of PROMs in clinical care may be helpful in identifying, discussing and eventually resolving aspects associated with HRQOL for these populations. Therefore, it is important to consolidate evidence on the use of PROMs and assess whether their implementation in clinical care can feasibly improve HRQOL and outcomes among children with chronic diseases.

\section{Healthcare utilisation}

Chronic diseases among children are associated with higher use of healthcare services including higher hospitalisation rates and length of stay in comparison to healthy children. ${ }^{23}{ }^{24}$ Evidence from adult patient populations suggests that the lower scores on PROMs were strongly associated with higher risk of death and hospitalisation. ${ }^{25}$ The use of PROMs is associated with improved symptom control and increased supportive care measures. ${ }^{6}$ Further, using PROMs in clinical care enables patients in the self-management of their long-term chronic conditions. ${ }^{26}$ For the parents of paediatric patients, caring for their hospitalised child often results in lost income and additional strain. ${ }^{22}$ This is in addition to the detrimental impacts hospitalisation can have on the social and economic status for the child in adulthood. ${ }^{27}{ }^{28}$ Considering the potential of PROMs to identify patients at greater risk for healthcare utilisation, ${ }^{7}$ it is essential to gather evidence regarding the role of PROMs on healthcare utilisation.

\section{Quality of care}

Researchers are increasingly utilising PROMs to assess performance of healthcare providers to improve the quality of care and patient satisfaction. ${ }^{29}$ PROMs can play an important role in providing patient-centred care by focusing on the patient's health goals and guiding therapeutic decisions. ${ }^{30}$ Healthcare systems have been incorporating advanced electronic platforms to support and simplify the implementation of PROMs in the clinical setting. ${ }^{18} 3132$ The use of PROMs data in an integrated manner would enable healthcare systems to orient evidence-based and patient-centred care. Evidence gathered through this systematic review will help healthcare systems to support quality improvement initiatives and develop effective strategies to further enhance quality of care.

\section{Objectives}

The objectives of our study are to: (1) identify previously implemented and evaluated generic or condition-specific self-reported PROMs for chronic conditions in paediatric settings; (2) consolidate the evidence to evaluate the impact of using PROMs on HRQOL, healthcare utilisation, patient outcomes (eg, symptoms control) and quality of care among paediatric patients with chronic conditions.

\section{METHODS AND ANALYSIS \\ Design}

This protocol was developed according to the Preferred Reporting Items for Systematic Review and Meta-Analysis Protocols(PRISMA-P) checklist, ${ }^{33}$ while the administration of the review and reporting will be carried out according to the PRISMA guidelines. ${ }^{34}$ The protocol for this review has been registered with PROSPERO, an international database of prospectively registered systematic reviews in health and social care.

\section{Patient and public involvement}

Patient-partners will be consulted to assess face validity of the included studies. The patient-partners are five individuals (three patients and two family-caregivers) that are members of the larger patient and family advisory group at the Alberta Children's Hospital. They will not be involved in developing research question, design and conduct of this review.

\section{Search strategy}

Our search strategy was developed according to the research question and guided by the study objectives. Keywords used for each key domains of the research question are as follows:

Population: Keywords 'child', 'adolescent' and 'pediatric care' will be used to identify studies focusing on paediatric populations (18 years or younger). 
Intervention: The interventions of interest for this review are PROMs, so keywords 'patient-reported outcomes', 'patient outcome assessment' and combination of 'outcome' and 'measures' along with the associated abbreviations (PRO, PROM) will be used to capture studies implementing these measures in clinical care.

Outcomes: The medical outcomes of interest for the systematic review are the impact of PROMs on HRQOL, healthcare utilisation, patient outcomes and quality of care. Keywords associated with the use of healthcare services including 'visits to emergency services', 'length of stay', 'patient admission' and 'patient readmission' nurse-patient relations' and 'physician-patient communication' are used to capture studies focusing on the overall utilisation of these services. Keywords associated with patient outcomes-'HRQoL', 'quality of life' and the indicators for the quality of healthcare will be used to build a robust search strategy which will include studies reporting these outcomes. The Boolean operator 'OR' will be used to combine terms within each outcome category. Finally, the Boolean operator 'AND' will be used to combine these concepts. English language filter will be applied on the final search results.

\section{Information sources}

We will systematically search MEDLINE (Ovid interface, 1950 onwards), EMBASE (Ovid Interface, 1974 onwards), CINAHL Plus with Full Text (EBSCOhost interface, 1982 onwards), PsycINFO (Ovid interface, 1803 onwards) and Cochrane Library (Ovid Interface, 1991 onwards). In addition to these electronic bibliographic databases, the reference lists of included studies will be searched in Web of Science (Thomson Reuters) database to ensure more complete coverage of the literature.

The search strategy for MEDLINE was developed iteratively with input from this systematic review team and support from a medical sciences librarian who has expertise in systematic review searching at the University of Calgary. As part of this iterative process, a primary search strategy was applied and 100 randomly chosen abstracts were reviewed by the systemic review team. These randomly chosen abstracts helped to specify the search strategy to ensure that it retrieves a high proportion of the eligible and key studies in this area. This revised search strategy was finalised after consulting again with the medical sciences librarian at the University of Calgary, and again after reviewing it with the senior researchers on the systemic review team with expertise in patient-oriented research.

Study design limits will not be imposed on the search. Implementation of PROMs in routine clinical practice mainly started after the year 2000 , so we will apply the time limit to exclude studies before the year 2000. Due to limited capacity in translating non-English articles, our literature search will be limited to the English language. Based on the recent evidence, ${ }^{35}$ we do not expect to introduce a systematic bias due the use of language restrictions in our systematic review. The MEDLINE strategy (see online supplementary appendix for the full MEDLINE [Ovid interface, 1950 onwards] search strategy) will be tested and adapted to the syntax of all other databases.

\section{Selection of studies}

Specific inclusion and exclusion criteria outlined below was developed after reviewing 100 randomly chosen abstracts by the members of the systematic review team (BM, $\mathrm{AC}$ and $\mathrm{SB}$ ) and through consultations with the librarian (DL) and senior researchers (M-JS and LH).

Inclusion criteria:

1. Studies including paediatric population and questionnaires completed by paediatric patients with chronic conditions (up to 18 years old).

2. Studies focusing primarily on the implementation and use of PROMs in paediatric chronic diseases.

3. Studies reporting primary data.

4. At least one of the following outcomes was reported: HRQOL, symptom control, mortality, healthcare utilisation, quality of care.

Exclusion criteria:

1. Studies reporting the use of PROMs for acute conditions, dental problems, pharmaceutical drug testing or surgical outcomes assessment.

2. Studies utilising secondary or retrospective data on PROMs.

3. Studies validating PROMs or testing methods for collecting/analysing PROMs.

4. Descriptive studies and reviews on PROMs to describe burden of disease and treatment

5. Studies reporting findings in languages other than English.

6. Studies published prior to the year 2000 .

\section{Data management}

Literature search results will be uploaded to EndNote Reference Management Software V.8. EndNote will be used to remove duplicate references, screen and manage all the references throughout the review process. A PRISMA flow diagram will be constructed to summarise the selection process.

\section{Selection process}

For the primary screening stage, titles and abstracts of the studies retrieved will be independently screened in duplicate by two reviewers working in pairs $(\mathrm{SB}, \mathrm{AC}$ and $\mathrm{BM})$ using the pre-determined eligibility criteria. This will reduce the potential for individual bias and the possibility of excluding relevant articles. Following the primary screening of the abstracts, full-text articles will be retrieved for studies meeting eligibility criteria or where titles or abstracts do not provide sufficient information to warrant their exclusion. Disagreements between reviewers will be resolved through discussion, and a third reviewer will be approached if the disagreement persists. Neither of the reviewers will be blind to the journal titles, study authors or the institutions. At the full-text review stage, reasons for excluding studies will be recorded. 


\section{Data extraction}

Two reviewers (SB and AC) will independently extract data from the included studies using a standardised data extraction form to reduce errors in data extraction. This form will be pilot tested by two reviewers (SB and AC). The data extraction form will include definitions of the variables to be extracted.

The following data will be extracted:

1. Summary data of included studies including author, year of publication, paediatric setting (tertiary, community care), location, patient population characteristics, chronic condition under study.

2. Type of PROM identified with descriptive statistics summarising general characteristics (Including name of the PROM, generic vs condition-specific and mode of administration, collection and reporting).

3. Reported outcome(s) of interest (Including impact on HRQOL, healthcare utilisation, patient outcomes and quality of care).

Extracted data from included studies will be presented to the whole research team to ensure consistency in data extraction. At this stage, patient partners will be consulted to verify if the extracted data is meaningful from the patients' perspective, ensuring that our study conforms to patient-oriented research. Consultation sessions will be organised with the patient-partners, where they will be briefed on the process of synthesising evidence through systematic review. The process and extracted data will be presented to them in lay terms. Then, face validity will be assessed by asking them if this systematic review measures what it purports to measure and if those findings make sense from patient's perspective.

\section{Data synthesis}

Finally, a PRISMA flow diagram will be presented to report the number of studies identified, screened and included in the final synthesis. Extracted data including participant characteristics, type of PROMs, geographical location, type of healthcare setting will be summarised in a table. Considering the scarcity of studies assessing the effectiveness of PROMs in routine clinical care of paediatric patients with chronic conditions, we do not anticipate conducting a subgroup analysis of generic versus condition-specific PROMs intervention. Post hoc analysis will be conducted to explore the effectiveness of PROMs on each outcome of interest. Narrative synthesis of the summarised data will be conducted to present the results of the review. Meta-analysis will be conducted using a random-effects model if there is sufficient homogeneity in terms of study design, type of intervention, comparators and outcomes among included studies. Publication bias will be assessed using funnel plot. Risk of bias in individual studies will be assessed independently by two reviewers ( $\mathrm{SB}$ and $\mathrm{AC}$ ) using the COnsensus-based Standards for the selection of health status Measurement INstruments (COSMIN) guideline for systematic reviews of PROMs. ${ }^{36}$ Assessing the methodological quality of included studies is important, but it is also recommended to assess the quality of PROMs included in the studies. ${ }^{36}$ This review might identify studies which used unvalidated PROMs, so we plan to use COSMIN checklist to assess the risk of bias for both methodological quality of studies and PROMs included in those studies. Discrepancies will be resolved by discussion and/or involvement of a third reviewer. In addition, the strength of body of evidence will be assessed as high, moderate or low using Grading of Recommendations Assessment, Development and Evaluation guidelines. ${ }^{37}$

\section{Dissemination}

The findings of this review will be disseminated through peer-reviewed publications, conference presentations and included in the lead author's doctoral dissertation.

\section{DISCUSSION}

Potential limitations of this study relate to the inability of predicting the strength of the evidence from the systematic review, however we will try to overcome this limitation by following a rigorous methodology and capitalise on our team members' expertise in knowledge synthesis.

This systematic review serves as a crucial step in the direction of integrating PROMs in paediatric clinical care. It will also reveal the extent to which PROMs were successful in affecting HRQOL, healthcare services utilisation, patient outcomes and quality of care for chronic diseases in paediatric population. Results of this review will guide healthcare policy and clinical care practices to incorporate paediatric patients' perspectives to deliver patient-centred care.

Acknowledgements The authors thank Lorraine Toews, Medical Sciences Librarian, University of Calgary for her assistance in development of the search strategy as detailed in the methods section of the protocol.

Contributors M-JS conceived the idea. SB drafted the manuscript. All authors provided comments on the manuscript. All authors contributed to the development of the selection criteria and data extraction criteria. SB, BM and AC developed the search strategy. M-JS and LH provided expertise on the use of patient-reported outcomes in clinical practice. All authors read, provided feedback and approved the final manuscript.

Funding This work was supported by the 2018 Innovation award from the Department of Pediatrics, University of Calgary.

Competing interests None declared.

Patient consent for publication Not required.

Provenance and peer review Not commissioned; externally peer reviewed.

Open access This is an open access article distributed in accordance with the Creative Commons Attribution Non Commercial (CC BY-NC 4.0) license, which permits others to distribute, remix, adapt, build upon this work non-commercially, and license their derivative works on different terms, provided the original work is properly cited, appropriate credit is given, any changes made indicated, and the use is non-commercial. See: http://creativecommons.org/licenses/by-nc/4.0/.

\section{REFERENCES}

1. Bai G, Herten MH, Landgraf JM, et al. Childhood chronic conditions and health-related quality of life: findings from a large populationbased study. PLoS One 2017;12:e0178539.

2. Karve S, Candrilli S, Kappelman MD, et al. Healthcare utilization and comorbidity burden among children and young adults in the United 
States with systemic lupus erythematosus or inflammatory bowel disease. J Pediatr 2012;161:662-70.

3. McPhail SM. Multimorbidity in chronic disease: impact on health care resources and costs. Risk Manag Healthc Policy 2016;9:143-56.

4. WHO. Noncommunicable Diseases, 2016. http://www.who.int/topics/ noncommunicable_diseases/en/

5. U.S. Department of Health and Human Services FDA Center for Drug Evaluation and Research, U.S. Department of Health and Human Services FDA Center for Biologics Evaluation and Research, U.S. Department of Health and Human Services FDA Center for Devices and Radiological Health. Guidance for industry: patientreported outcome measures: use in medical product development to support labeling claims: draft guidance. Health Qual Life Outcomes 2006;4:79.

6. Kotronoulas G, Kearney N, Maguire R, et al. What is the value of the routine use of patient-reported outcome measures toward improvement of patient outcomes, processes of care, and health service outcomes in cancer care? A systematic review of controlled trials. J Clin Oncol 2014;32:1480-501.

7. Hinami K, Smith J, Deamant CD, et al. When do patient-reported outcome measures inform readmission risk? J Hosp Med 2015;10:294-300.

8. El Miedany Y, El Gaafary M, El Arousy N, et al. Arthritis education: the integration of patient-reported outcome measures and patient selfmanagement. Clin Exp Rheumatol 2012;30:899-904.

9. Santana MJ, Feeny D. Framework to assess the effects of using patient-reported outcome measures in chronic care management. Qual Life Res 2014;23:1505-13.

10. Basch E, Deal AM, Dueck AC, et al. Overall Survival Results of a Trial Assessing Patient-Reported Outcomes for Symptom Monitoring During Routine Cancer Treatment. JAMA 2017;318:197-8.

11. Brocklehurst P, Price J, Glenny AM, et al. The effect of different methods of remuneration on the behaviour of primary care dentists. Cochrane Database Syst Rev 2013;11:CD009853.

12. Santana MJ, Feeny D, Johnson JA, et al. Assessing the use of health-related quality of life measures in the routine clinical care of lung-transplant patients. Qual Life Res 2010;19:371-9.

13. Santana MJ, Feeny DH. Using the health utilities index in routine clinical care: process, feasibility, and acceptability: a randomized controlled trial. Patient 2009;2:159-67.

14. Weldring T, Smith SM. Patient-Reported Outcomes (PROs) and Patient-Reported Outcome Measures (PROMs). Health Serv Insights 2013;6:61-8.

15. Calvert MJ, Freemantle N. Use of health-related quality of life in prescribing research. Part 1: why evaluate health-related quality of life? J Clin Pharm Ther 2003;28:513-21.

16. Kyte DG, Calvert M, van der Wees PJ, et al. An introduction to patient-reported outcome measures (PROMs) in physiotherapy. Physiotherapy 2015;101:119-25.

17. Bevans KB, Moon J, Carle AC, et al. Patient reported outcomes as indicators of pediatric health care quality. Acad Pediatr 2014;14(5 Suppl):S90-S96.

18. Haverman L, van Rossum MA, van Veenendaal M, et al. Effectiveness of a web-based application to monitor health-related quality of life. Pediatrics 2013;131:e533-e543.

19. Engelen V, Haverman L, Koopman $\mathrm{H}$, et al. Development and implementation of a patient reported outcome intervention (QLIC-ON
PROfile) in clinical paediatric oncology practice. Patient Educ Couns 2010;81:235-44.

20. Haverman L, Engelen V, van Rossum MA, et al. Monitoring healthrelated quality of life in paediatric practice: development of an innovative web-based application. BMC Pediatr 2011;11:3.

21. WHOQOL: Measuring Quality of Life. Health statistics and information systems. Available: http://www.who.int/healthinfo/survey/ whoqol-qualityoflife/en/ (cited 05 Sep 2018).

22. LeBlanc LA, Goldsmith T, Patel DR. Behavioral aspects of chronic illness in children and adolescents. Pediatr Clin North Am 2003;50:859-78.

23. Pao M, Ballard ED, Rosenstein DL. Growing up in the hospital. JAMA 2007:297:2752-5.

24. Newacheck PW, Halfon N. Prevalence and impact of disabling chronic conditions in childhood. Am J Public Health 1998;88:610-7.

25. Mapes DL, Lopes AA, Satayathum S, et al. Health-related quality of life as a predictor of mortality and hospitalization: the Dialysis Outcomes and Practice Patterns Study (DOPPS). Kidney Int 2003;64:339-49.

26. Greenhalgh J, Dalkin S, Gooding K, et al. Functionality and feedback: a realist synthesis of the collation, interpretation and utilisation of patient-reported outcome measures data to improve patient care. Southampton (UK): NIHR Journals Library, 2017.

27. Miedema B, Easley J, Fortin P, et al. The economic impact on families when a child is diagnosed with cancer. Curr Oncol 2008;15:173-8.

28. Didsbury MS, Kim S, Medway MM, et al. Socio-economic status and quality of life in children with chronic disease: a systematic review. $J$ Paediatr Child Health 2016;52:1062-9.

29. Schlesinger M, Grob R, Shaller D. Using patient-reported information to improve clinical practice. Health Serv Res 2015;50(Suppl 2):2116-54.

30. Van Der Wees PJ, Nijhuis-Van Der Sanden MW, Ayanian JZ, et al Integrating the use of patient-reported outcomes for both clinical practice and performance measurement: views of experts from 3 countries. Milbank Q 2014;92:754-75.

31. Bouazza YB, Chiairi I, El Kharbouchi O, et al. Patient-reported outcome measures (PROMs) in the management of lung cancer: A systematic review. Lung Cancer 2017;113:140-51.

32. Jensen RE, Snyder CF, Abernethy AP, et al. Review of electronic patient-reported outcomes systems used in cancer clinical care. $J$ Oncol Pract 2014;10:e215-e222.

33. Shamseer L, Moher D, Clarke M, et al. Preferred reporting items for systematic review and meta-analysis protocols (PRISMA-P) 2015: elaboration and explanation. BMJ 2015;350:g7647.

34. Moher D, Liberati A, Tetzlaff J, et al. Preferred reporting items for systematic reviews and meta-analyses: the PRISMA statement. PLOS Med 2009;6:e1000097.

35. Morrison A, Polisena J, Husereau D, et al. The effect of Englishlanguage restriction on systematic review-based meta-analyses: a systematic review of empirical studies. Int $\mathrm{J}$ Technol Assess Health Care 2012;28:138-44.

36. Prinsen CAC, Mokkink LB, Bouter LM, et al. COSMIN guideline for systematic reviews of patient-reported outcome measures. Qual Life Res 2018;27:1147-57.

37. Guyatt G, Oxman AD, Akl EA, et al. GRADE guidelines: 1 . Introduction-GRADE evidence profiles and summary of findings tables. J Clin Epidemiol 2011;64:383-94. 